\title{
Foraminal stenosis complicating retained broken epidural needle tip -A case report-
}

\author{
Ji Wong You, and Yong-Hyun Cho \\ Department of Anesthesiology and Pain Medicine, Seoul Secred Heart Hospital, Seoul, Korea
}

Lumbar epidural anesthesia is useful in a variety of chronic benign pain syndromes, including lumbar radiculopathy, low back pain syndrome, spinal stenosis, and vertebral compression fractures. Given the increased number of epidural nerve blocks being performed, some have reported unexplained complications of a transient or permanent nature and with varying degrees of severity. However, no case has been reported of a broken epidural needle tip retained in the lumbar facet joint area. This represents the first reported case presentation of foraminal stenosis developing in a patient after a retained epidural needle tip. (Korean J Anesthesiol 2010; 59: S69-S72)

Key Words: Broken epidural needle tip, Epidural anesthesia, Spinal stenosis.

Since epidural block anesthesia is able to shut out the pain channel in a relatively safe and selective way, it is widely performed for the diagnosis and treatment of acute and chronic pains. Recently, the lumbar approach for steroid injection has been increasingly performed for various cases including patients [1] with lumbar radiculopathy, low back pain syndrome, foraminal stenosis, post laminectomy syndrome, phantom pain, diabetic polyneuropathy, and vertebral compression fracture. Complications of epidural block anesthesia include headache from dural fracture, backache, spinal injury, epidural haematoma, epidural abscess from injection, meningitis, and osteomyelitis, but have low rates of occurrence.

The authors report the case of a patient with backache who underwent surgical foreign body removal and posterior lumbar interbody fusion due to spinal stenosis retained from a broken epidural needle tip, which was stuck in the facet joint during epidural block anesthesia. This report also includes a literature review.

\section{Case Report}

The 39-year-old female patient visited the hospital as an outpatient, complaining of pains from backache and the right leg. The patient had a lumbar MRI scan for back pain at another hospital three years ago, but there was no unusual diagnosis. For pain control, an epidural nerve block was performed at another hospital, but the symptom had not improved yet. After the operation, the patient gradually got pains at the back and right leg, and the symptom became worse starting a year ago. A physical examination showed pains during the silent

Received: April 28, 2010. Revised: 1st, May 14, 2010; 2nd, May 26, 2010. Accepted: June 16, 2010.

Corresponding author: Yong-Hyun Cho, M.D., Department of Anesthesiology and Pain Medicine, Seoul Secred Heart Hospital, Cheongnyangnidong, Dongdaemun-gu, Seoul 130-010, Korea. Tel: 82-2-966-1616, Fax: 82-2-968-2394, E-mail: anesthecho@naver.com

(c) This is an open-access article distributed under the terms of the Creative Commons Attribution Non-Commercial License (http:// creativecommons.org/licenses/by-nc/3.0/), which permits unrestricted non-commercial use, distribution, and reproduction in any medium, provided the original work is properly cited. 
period, referred pain to the right leg, and radiating pain from the lumbar $5^{\text {th }}$ segment. From the straight leg raising test, the patient complained of pains from both legs at an 80 degree angle. However, any disorder or decompression of motor nerve and sensory nerve was not detected. The lumbar X-ray picture scanned on the same day showed a small metal foreign body (Fig. 1).

With the MRI was scanned at the hospital, doctors diagnosed degenerative spondyloisthesis at the lumbar $4-5^{\text {th }}$ segment, and degenerative change from the facet joint as well as a foreign body (Fig. 2). The hospital diagnosed spinal stenosis and a foreign body at the facet joint, and decided to perform posterior lumbar interbody fusion and foreign body removal from the right facet joint of the lumbar $4-5^{\text {th }}$ segment.

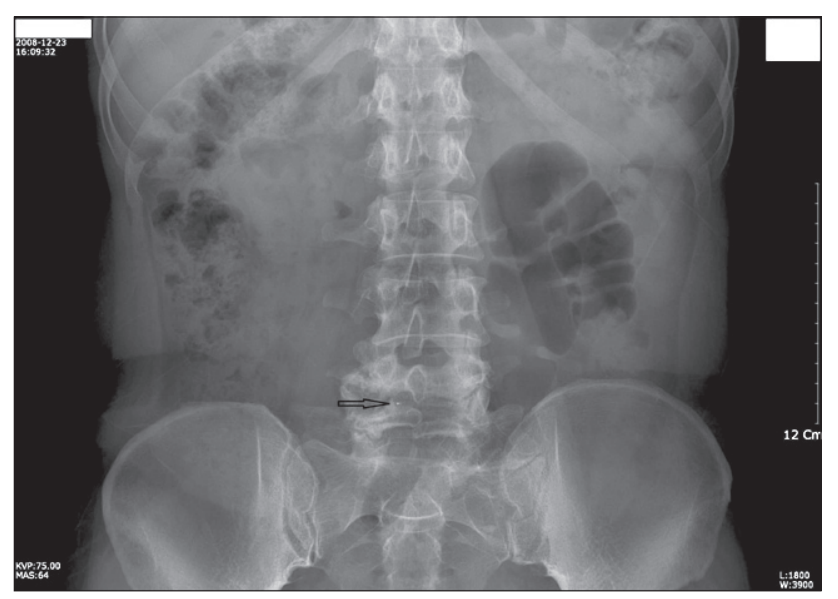

Fig. 1. Lumbar spine X-ray image shows metallic foreign body in right lumbar 4, 5 spine facet joint area.

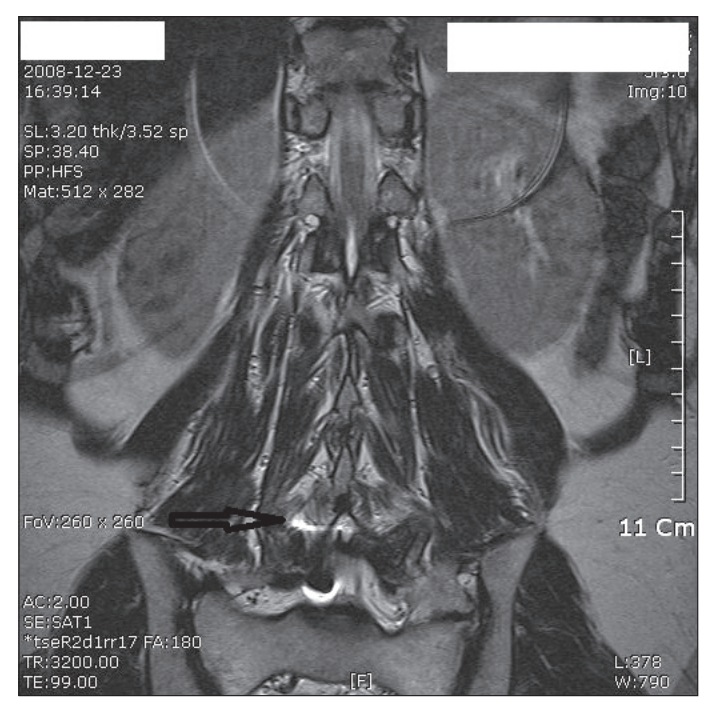

Fig. 2. Lumbar MRI image in sagital view shows metallic material in lumbar spine area.
The patient was $158 \mathrm{~cm}$ tall and weighed $62 \mathrm{~kg}$. Her liver function examination was normal before operation, except it was positive for the antibody for hepatitis C. Except that, there was no special diagnosis before operation. For the treatment before anesthesia, glycopyrrolate $0.2 \mathrm{mg}$ was injected into the muscle on the very day of operation. After arriving at the operation room, $18 \mathrm{G}$ intravenous route was additionally secured at the right side of the upper arm, and an ECG, a noninvasive blood pressure monitor, pulse oximetry, and capnogram were established. After identifying stable blood pressure and pulse rates, 100\% oxygen $6 \mathrm{~L} / \mathrm{min}$ was provided through a mask for 1-2 minutes. For the induction of anesthesia, propofol $2 \mathrm{mg} / \mathrm{kg}$ was injected into the muscle for 20 seconds, and after identifying the loss of lid reflex and the stability of blood pressure and pulse rates, rocuronium 0.9 $\mathrm{mg} / \mathrm{kg}$ was injected into the muscle. After sufficient muscle relaxation was identified after forced breathing with the mask was performed for 2 minutes when there was no spontaneous breathing, intubation was performed. For the maintenance of anesthesia, oxygen $2 \mathrm{~L} / \mathrm{min}$, nitrous oxide $2 \mathrm{~L} / \mathrm{min}$, and isoflurane $1.5-2.0 \mathrm{vol} \%$ were used. With the favorable vital signs of the patient, the posture was changed to the prone position, and the surgery was performed after finishing all necessary preparations. The operating surgeon removed the metal foreign body stuck in the right side of the facet joint at the lumbar $4-5^{\text {th }}$ segment, and finished the planned posterior lumbar interbody fusion safely. After the operation, the patient recovered well and left the hospital after one week. The foreign body removed from the operation was a stuffed needle with a sloping side made from a metal in silver color; its diameter was less than $1 \mathrm{~mm}$ and its length was $0.7 \mathrm{~cm}$ (Fig. 3). Based on the previous epidural

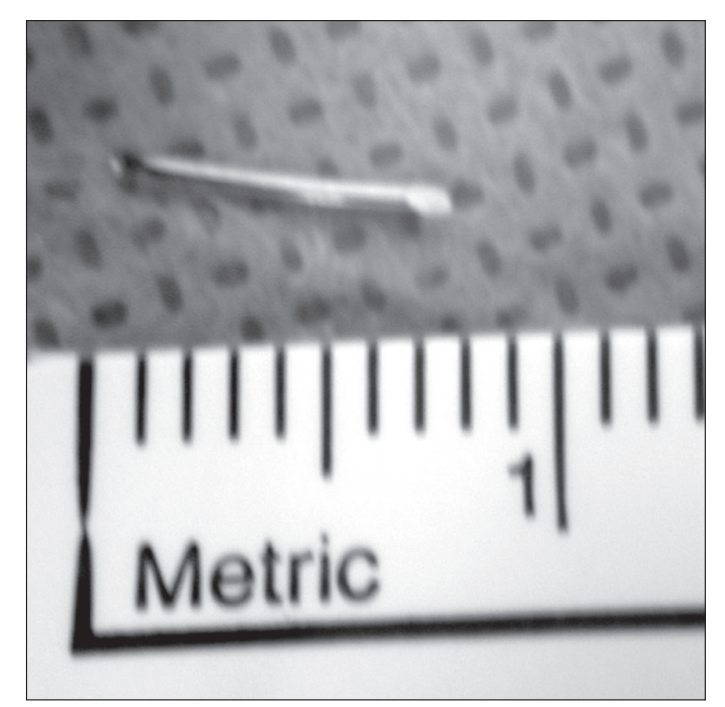

Fig. 3. Removed foreign body shows needle like shape and beveled tip. 
block history of the patient and the shape of the foreign body, it is considered to be the broken stylet of an epidural needle tip.

\section{Disccusion}

Epidural block anesthesia is performed not only for anesthesia of surgical operations but also for pain control after operations and treatment of chronic pains. It is known that its complications rarely occur, but haematoma and spinal nerves injuries, as well as neuromuscular injuries, might break out.

Staats et al. [2] once reported that spinal stenosis occurred due to a reactive epidural clot surrounding a catheter fragment which was cut by $1.5 \mathrm{~cm}$ in the process of catheter insertion and retained inside the epidural space; after removing the catheter fragment and injured tissue, symptoms of the patients were improved. Bonica et al. [3] reported 3 cases of retaining epidural catheter fragments inside the epidural space. Reports say that 2 of them required surgical removals while the other did not because no complication was detected.

Collier [4] described that due to the recent technology development, it is hard to damage an epidural catheter and an epidural block anesthesia needle tip can be broken only with power more than $1-2 \mathrm{~kg}$. Accordingly, they argued that main culprit of damage is a defect in the process of manufacturing or mistakes of the operating surgeon. Abou-Shameh et al. [5] reported the case that although a catheter was successfully inserted during a spinal-epidural joint block, a dural puncture was difficult because the needle for spinal anesthesia was short due to the condition of extreme obesity. In that case, the operating surgeon tried spinal anesthesia at the lumbar space one segment lower, but after a number of trials, the spinal anesthesia needle was removed. After the removal, the distant part of the needle disappeared by about $5 \mathrm{~cm}$, and the cut needle tip was removed by performing surgical operation. They reported that the case of the broken needle tip occurred twice in 20 years, showing an occurrence rate of 1: 5,000.

Lumbar spinal stenosis is the disease in which the narrowed spine presses the spinal cord. According to Kirkaldy-Willis et al. [5], facet joint suffers facet joint syndrome and synovitis from early hypofunction, adding more articular capsules, and the joint becomes unstable so that the vertebral body transfers to the front. Repeated stimulus from instability after the transmission causes thickening and osteophyte formation restabilizing spinal segments. However, both thickened structures at the rear and bulging or projecting intervertebral disc at the front suppress the driving region of the nerves, causing spinal stenosis [6].

Mostly, the cause of spinal stenosis is a degenerative change of the spine due to aging. For a congenital cause, achondroplasia leads to severe stenosis due to a short pedicle, bulged facet joint, and lamina, and this becomes a cause of early spinal stenosis. Also, the spinal stenosis can lead to the second transmutation of spinal stenosis [6]. Among secondary causes, iatrogenic stenosis can occur after laminectomy or spinal fusion. Moreover, as mentioned earlier, there is a case reported about spinal stenosis occurring by a foreign body inside the vertebral space.

The patient in this case is 39 years old, so it is unlikely to be a degenerative spinal stenosis from aging. Moreover, while there is no special diagnosis for other spinal segments, an MRI scan of the right facet joint in which the needle tip was detected showed a second transmutation including degenerative changes and thickening of the neighboring ligamentum flavum. Against the backdrop, it is assumed that the patient had a retained needle tip broken during an epidural block procedure, then the second transmutation occurred around the right facet joint, leading to spinal stenosis.

A broken needle tip can occur from a manufacturing problem, rather than from operation techniques. For an ideal needle for epidural block, Collier [4] argue that it should be sufficiently sharp to cut spinal ligaments and simultaneously be sufficiently dull so as to not perforate the dural. Chin et al. [7] reported the case when the needle tip and hub of spinal anesthesia injection detached from each other. They assumed that careless manipulation, repeated usages of the spinal needle, and the obesity of the patient were the main culprits, and insisted that an epidural block should be carefully operated under accurate knowledge of anatomy. When facing resistance during the insertion of a needle or catheter for epidural block anesthesia, immoderate proceeds or giving excessive power should be prohibited. Also, after finishing an operation, the needle should be checked. The case of spinal stenosis due to the fracture of an epidural block catheter retained inside the epidural space occurred when the tip of the catheter was cut during pulling the catheter back because of resistance in the process of insertion [2]. Chin et al. [7] also highlighted the careful operation of epidural block anesthesia. Blanchard et al. [8] described the case where a patient complaining of radiating pain required a surgical operation due to a retained catheter during the insertion process of a catheter for epidural block anesthesia. Blass et al. [9] introduced the case where the retained catheter was removed through lumbar laminectomy since it was impossible to remove it manually because the catheter was stuck and twisted around the ligamentum flavum. In most cases, however, a catheter being retained inside the epidural space does not cause problems [10]. If part of the catheter during epidural block anesthesia is retained inside patients, it is not normally removed. For rare cases accompanying neurologic symptoms or second transmutations, surgical operations might be required.

When operating epidural block anesthesia, accurate know- 
ledge of anatomy should be a prerequisite to avoid careless manipulation. Also, operators should check whether the tip of needle or catheter is broken or not with the naked eye, and when the retained fracture is doubtful, they should consider X-ray scanning. So far, there have been reports about spinal stenosis due to retained fracture of a catheter inside the vertebral space. However, there is none about the case describing spinal stenosis from retained catheter fracture inside the spinal space. Therefore, it is considered that additional data collection and monitoring are required.

\section{References}

1. Waldman SD. Interventional pain management. 2nd ed. Philadelphia, WB Saunders company. 2001, pp 415-22.

2. Staats PS, Stinson MS, Lee RR. Lumbar stenosis complicating retained epidural catheter tip. Anesthesiology 1995; 83: 1115-8.

3. Bonica JJ, Backup PH, Anderson CE, Hadfield D, Crepps WF,
Monk BF. Peridural block: analysis of 3,637 cases and a review. Anesthesiology 1957; 18: 723-84

4. Collier C. Epidural catheter breakage: a possible mechanism. Int J Obstet Anesth 2000; 9: 87-93.

5. Abou-Shameh MA, Lyons G, Roa A, Mushtaque S. Broken needle complicating spinal anesthesia. Int J Obstet Anesth 2006; 15: 178-9.

6. Kirkaldy-Willis WH, Farfan HF. Instability of the lumbar spine. Clin Orthop Relat Res 1982; 165: 110-23.

7. Chin JH, Jun IG, Kim YK, Kang KN, Hwang GS, Hwang JH. Inadvertent breakage of spinal needle during the obturator nerve block for the transurethral resection of bladder tumor. Korean J Anesthesiol 2007; 53: 250-3.

8. Blanchard N, Clabeau JJ, Ossart M, Dekens J, Legars D, Tchaoussoff J. Radicular pain due to a retained fragment of epidural catheter. Anesthesiology 1997; 87: 1567-9.

9. Blass NH, Roberts RB, Wiley JK. The case of the errant epidural catheter. Anesthesiology 1981; 54: 419-21.

10. Bromage PR. Epidural analgesia. Philadelphia, WB Saunders. 1978 pp 664-5. 